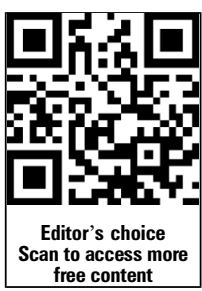

For numbered affiliations see end of article.

\section{Correspondence to} Professor John T O'Brien, Department of Psychiatry, University of Cambridge, Cambridgeshire and Peterborough NHS Foundation Trust, Box 189, Level E4 Cambridge Biomedical Campus, Cambridge, CB2 OSP, UK; john.obrien@medschl.cam. ac.uk

Received 22 July 2013 Revised 9 April 2014 Accepted 23 April 2014 Published Online First 23 May 2014

\section{CrossMark}

\section{To cite: Gore $\mathrm{RL}$, Vardy ERLC, O'Brien JT. \\ J Neurol Neurosurg \\ Psychiatry 2015:86:50-59.}

\title{
Delirium and dementia with Lewy bodies: distinct diagnoses or part of the same spectrum?
}

\author{
Rachel L Gore, ${ }^{1,2}$ Emma R L C Vardy, ${ }^{1,3}$ John T O'Brien ${ }^{1,4}$
}

ABSTRACT

Dementia with Lewy bodies (DLB) is recognised as the second most common form of dementia in older people. Delirium is a condition of acute brain dysfunction for which a pre-existing diagnosis of dementia is a risk factor. Conversely delirium is associated with an increased risk of developing dementia. The reasons for this bidirectional relationship are not well understood. Our aim was to review possible similarities in the clinical presentation and pathophysiology between delirium and $D L B$, and explore possible links between these diagnoses. A systematic search using Medline, Embase and Psychinfo was performed. References were scanned for relevant articles, supplemented by articles identified from reference lists and those known to the authors. 94 articles were selected for inclusion in the review. Delirium and DLB share a number of clinical similarities, including global impairment of cognition, fluctuations in attention and perceptual abnormalities. Delirium is a frequent presenting feature of DLB. In terms of pathophysiological mechanisms, cholinergic dysfunction and genetics may provide a common link. Neuroimaging studies suggest a brain vulnerability in delirium which may also occur in dementia. The basal ganglia, which play a key role in DLB, have also been implicated in delirium. The role of Cerebrospinal fluid (CSF) and serum biomarkers for both diagnoses is an interesting area although some results are conflicting and further work in this area is needed. Delirium and DLB share a number of features and we hypothesise that delirium may, in some cases, represent early or 'prodromal' DLB. Further research is needed to test the novel hypothesis that delirium may be an early marker for future DLB, which would aid early diagnosis of DLB and identify those at high risk.

\section{INTRODUCTION}

Dementia is an increasing burden on our society with the ageing population. There are currently over 800000 people in the UK alone with dementia and around 30 million worldwide, with rates doubling in the next 30 years. ${ }^{1}$ One of the earliest reports of dementia with Lewy bodies (DLB) was by Kosaka et al who reported the finding of 'Lewy bodies' throughout the cortex of some patients with dementia. ${ }^{2}$ DLB is now recognised as the one of the most common forms of dementia in older people accounting for around $15-20 \%$ cases at autopsy. ${ }^{3}{ }^{4} \mathrm{~A}$ recent systematic review shows that the prevalence in the community setting is $4.2 \%$, increasing to $7.5 \%$ in secondary care, ${ }^{5}$ suggesting that DLB is not currently diagnosed as frequently as would be expected from autopsy studies.
The International Consensus Criteria for $\mathrm{DLB}^{6}$ are the accepted standard for clinical and pathological diagnosis of DLB. Probable DLB can be diagnosed clinically in the presence of progressive cognitive decline accompanied by two out of three core features; fluctuating cognition, recurrent visual hallucinations and spontaneous motor parkinsonism. ${ }^{6}$ Suggestive features include rapid eye movement sleep behaviour disorder (RBD), severe neuroleptic sensitivity and low dopamine transporter uptake in the basal ganglia demonstrated by single photon emission CT (SPECT) or positron emission tomography imaging. ${ }^{6}$ In studies using postmortem diagnosis of DLB as the gold standard, a clinical diagnosis of DLB can be made with a sensitivity of $78-83 \%$ and a specificity of $85-95 \%$ in expert centres, though many sites report sensitivity of $50 \%$ or less. $^{7}$

Delirium is a complex neuropsychiatric condition of acute brain dysfunction with multifactorial aetiology ${ }^{8}$ Population prevalence in those aged $65+$ years is $1-2 \%$, with rates rising to $10 \%$ in those aged $85+$ years, and up to $22 \%$ in populations with higher prevalence of dementia. ${ }^{9}$ Delirium is common in hospitalised older patients with prevalence at admission between $10 \%$ and $31 \%$ and incidence rates between $11 \%$ and $42 \% .{ }^{10}$ Presentation of delirium is highly variable; Disturbances in attention and awareness are central to the diagnosis, ${ }^{11}$ with other common abnormalities including abnormal motor behaviour, sleep wake cycle abnormalities and disturbances in emotion, perception and thinking. ${ }^{8}$ Abnormalities in motor behaviour form the basis for the categorisation of delirium into three subtypes; hyperactive, hypoactive and mixed delirium. ${ }^{12}$ The diagnosis of delirium can be aided by use of diagnostic tools and rating scales, including the Confusion Assessment Method, a diagnostic instrument for delirium based on the Diagnostic and Statistical Manual of Mental Disorders (DSM) III-R diagnostic criteria. ${ }^{13}$ DSM $\mathrm{V}$ and International Classification of Disease $10 \mathrm{can}$ also be used to aid the diagnosis. ${ }^{11}{ }^{14}$ The DSM V criteria is listed in box $1 .^{11}$

Delirium is much more common in patients with dementia or chronic cognitive impairment (OR 5.3). ${ }^{15}$ It has a number of causes including infection, stroke, metabolic imbalance, substance misuse and withdrawal, and systemic illness of almost any type, especially when associated with pyrexia. As such, a full investigative screen, which may include the need for structural brain imaging, is indicated. Delirium is associated with increased morbidity, mortality and longer hospital stays, ${ }^{16}$ 
Box 1 DSM-V criteria for a diagnosis of Delirium

A. Disturbance in attention (ie, reduced ability to direct, focus, sustain and shift attention) and awareness (reduced orientation to the environment).

B. The disturbance develops over a short period of time (usually hours to a few days), represents a change from baseline attention and awareness, and tends to fluctuate in severity during the course of a day.

C. An additional disturbance in cognition (eg, memory deficit, disorientation, language, visuospatial ability or perception).

D. The disturbances in Criteria $A$ and $C$ are not better explained by another pre-existing, established or evolving neurocognitive disorder and do not occur in the context of a severely reduced level of arousal, such as coma.

E. There is evidence from the history, physical examination or laboratory findings that the disturbance is a direct physiological consequence of another medical condition, substance intoxication or withdrawal (ie, due to a drug of abuse or to a medication), or exposure to a toxin, or is due to multiple aetiologies.

DSM, Diagnostic and Statistical Manual.

with reported death rate at discharge of $14-37 \% .{ }^{10}$ Delirium is also associated with subsequent cognitive decline and increases the risk of dementia (OR 8.7, 95\% CI 2.1 to 35$)^{17} 18$ and worsens dementia severity in subjects with a pre-existing diagnosis (OR 3.1, 95\% CI 1.5 to 6.3). ${ }^{19}$

Despite this evidence, there is little published data regarding whether a particular type of dementia has a stronger association with delirium than others and more specifically whether delirium may represent the prodromal phase of any particular type of dementia. Two small studies are notable; One study conducted in Finland in the mid-1990s found that the majority of patients who developed dementia after an episode of delirium were diagnosed with Alzheimer's Disease (AD). ${ }^{20}$ The same group published a population based study of 199 subjects, and showed an episode of delirium to be associated with dementia, more specifically vascular dementia $(\mathrm{VaD}) .{ }^{21}$ The timing of the studies and the development of the 1996 DLB consensus criteria ${ }^{6}$ may have impacted on the clinical diagnosis rate of DLB in these studies. It is notable from clinical practice that some of the features of delirium overlap with DLB. We hypothesise that there may be a link between delirium and DLB, and that delirium may represent a prodrome or early stage of DLB, representing a diagnostic opportunity for DLB. A literature review was therefore undertaken to explore the possible links between DLB and delirium further and to review similarities or otherwise between these two diagnoses.

\section{METHODS}

A literature search using Medline, Embase and Psychinfo was performed in July 2013, and repeated in March 2014 covering the period from 1984 to the present using the keywords "dementia with lewy bodies" OR "lewy body dementia" OR "DLB", and "delirium" OR "acute confusional state". Combining "dementia with lewy bodies" OR "lewy body dementia" OR "DLB” AND "delirium" OR "acute confusional state" yielded 137 articles. The abstracts of these articles were read and full texts of those relevant to the review were obtained. The decision was made to combine the DLB and delirium search terms. This may not have been as exhaustive as searching for DLB and delirium separately, however since the main focus of the article was to explore the possible links between these two diagnoses, it was felt that this search would yield the most relevant articles for the review. In order to ensure that all relevant references were sourced, references were scanned for other relevant articles, supplemented by articles known to the authors. Given the breadth of areas covered in this review, a further literature search was conducted to find more specific literature on the subheadings of this review including using the search terms "Neuroimaging", "Biomarkers" and "Neurobiology" and combining these with the relevant DLB and delirium search terms at this stage. A total of 94 articles were incorporated into the review.

\section{Clinical presentation}

Onset and course

The onset of the cognitive impairment in DLB is insidious ${ }^{22}$ with a progressive decline over years, whereas delirium is a reversible state of brain dysfunction with acute onset. ${ }^{23}$ The average length of a delirium episode is reported as between 3 days and 13 days, ${ }^{24}$ though it may persist for weeks to months in some patients. ${ }^{25}$ This 'prolonged delirium' may be difficult to distinguish from DLB.

\section{Cognitive impairment}

The central feature required for the diagnosis of DLB is progressive cognitive decline of sufficient magnitude to interfere with normal social and occupational functioning. ${ }^{26}$ Those with DLB are more impaired than $\mathrm{AD}$ subjects in cognitive domains including visual tracking, visual attention shifting, working memory and visuospatial skills (table 1). ${ }^{27}$ This is similar to delirium, where deficits in attention, short-term memory and visuospatial deficits can be particularly marked. ${ }^{28} 29$ One study reported that mean visuospatial ability scores using the delirium rating scale were almost as impaired as attention, which may explain some of the common features of delirium including visual perceptual abnormalities and underlying problems such as wandering. ${ }^{29}$ Other studies report that specific deficits in visual perception have been described in delirium, however this may not necessarily be related to cognitive performance. ${ }^{30}$ Visual perception abnormalities, namely visual hallucinations, are a core feature of DLB. ${ }^{6}$

\section{Fluctuating cognition and consciousness}

Fluctuations in cognitive function occur in $58 \%$ of those with DLB at the time of presentation and in $75 \%$ of cases at some point during the course of the illness. ${ }^{26}$ Although fluctuations can occur in most dementias, patients with DLB have a greater prevalence and severity of fluctuating cognition compared with $\mathrm{AD}$ or $\mathrm{VaD}$, rated using clinical, attentional and EEG measures. ${ }^{31}$ The clinician assessment of fluctuation (CAF) is a tool to measure such fluctuation. ${ }^{32}$ Fluctuations in consciousness may manifest in DLB as changes in the level of arousal and excessive daytime drowsiness. Delirium has been described as 'fundamentally a disorder of consciousness', with clouding of consciousness (state of reduced wakefulness or awareness that may include hyperexcitability and irritability alternating with drowsiness) being a core feature of delirium and occurring in $65-100 \%$ of cases. $^{33}$ The fluctuations in consciousness seen in delirium can be understood in terms of arousal and its relation to circadian rhythms. ${ }^{33}$

It is such fluctuations seen in DLB and delirium that make it difficult to distinguish these two diagnoses, and may represent a diagnostic challenge. Although fluctuations form part of the 
Table 1 Cognitive deficits in DLB and delirium ${ }^{27-30}$

\begin{tabular}{|c|c|c|c|}
\hline $\begin{array}{l}\text { Cognitive deficits in DLB } \\
(D L B<A D)\end{array}$ & Rating scale or neuropsychological test & Cognitive deficits in delirium & $\begin{array}{l}\text { Rating scale or } \\
\text { neuropsychological test }\end{array}$ \\
\hline Visual tracking & Trail making $A$ & Orientation & ACE-R \\
\hline Visual attention shifting & Trail making B & Attention & CTD \\
\hline Working memory & Digit span & Memory & CTD \\
\hline Executive function & $\begin{array}{l}\text { Similarities Initiation/perseveration subtest } \\
\text { of the MDRS }\end{array}$ & Vigilance & CTD \\
\hline Verbal fluency/language & Lexical fluency & Language & DRS \\
\hline Visuospatial functioning & $\begin{array}{l}\text { Block design (WAIS) } \\
\text { Clock (copy) } \\
\text { Cube copy (ACE-R) } \\
\text { Construction (MDRS) }\end{array}$ & $\begin{array}{l}\text { Visuospatial functioning and visual } \\
\text { perception }\end{array}$ & $\begin{array}{l}\text { DRS } \\
\text { Clock (copy) } \\
\text { Cube copy (ACE-R) } \\
\text { Visual object and space perception } \\
\text { battery }\end{array}$ \\
\hline
\end{tabular}

core diagnostic criteria for DLB and are common in delirium, it is a feature which can be difficult to identify since there are no diagnostic tools which quantify and distinguish the aetiology of such fluctuations. In DLB, the tool presently applied to identify fluctuating cognition, the CAF questionnaire, lacks sensitivity; the CAF was proposed as a reliable diagnostic tools for patients with DLB with a moderate dementia, but not those at the earliest stage of their disease. ${ }^{32}$ The development of additional tools would be welcome.

\section{Perceptual disturbances and delusions}

Psychotic symptoms occur in around $80 \%$ of patients with DLB. ${ }^{34}$ Visual hallucinations are present at the time of presentation of DLB in 33\% of cases and occur during the course of the illness in around half of cases. ${ }^{35}$ Delusions are common in DLB, occurring in $56 \%$ at the time of presentation and $65 \%$ at some point during the illness. ${ }^{35}$ Psychotic features are also present in delirium and complicate around half of cases. ${ }^{8}$ Similar to DLB, these psychotic features tend to consist of visual hallucinations predominately, but illusions and delusions also occur. Psychotic features tend to occur in the hyperactive delirium subtype. ${ }^{12}$

\section{Motor disturbances}

Spontaneous motor features of parkinsonism, or extrapyramidal symptoms (EPS), are a core feature of $\mathrm{DLB}^{6}$ reported in 25$50 \%$ of patients with DLB at the time of diagnosis. Most patients will develop some EPS during the course of the disease. ${ }^{26}$ Motor disturbances are frequently present in delirium, however they are dissimilar to those occurring in DLB and include features of overactivity such as agitation, motor restlessness or underactivity such as motor retardation. ${ }^{12}$ It is these motor disturbances in delirium which give rise to the delirium subtypes 'hyperactive', 'hypoactive' and 'mixed' delirium. ${ }^{36}$

\section{Falls and syncope}

Approximately a third of patients with DLB experience repeated falls and syncope. ${ }^{37}$ Postural instability contributes to the high frequency of falls in DLB. Delirium is also associated with an increased risk of falling; one study looking at fallers in hospital showing that $96 \%$ had evidence of a delirium. ${ }^{38}$ The reasoning behind this increased risk of falls in delirium is likely to be multifactorial and related to the delirium itself (disorientation and psychomotor abnormalities) and the prescription of medications to treat some of the symptoms of delirium which may have a sedative effect, for example benzodiazepines and antipsychotics. ${ }^{39}$

\section{Sleep disturbances}

DLB is associated with sleep disturbances, namely RBD. ${ }^{6}$ RBD can present a number of years before the onset of other DLB symptoms, and a role as a marker for underling DLB has been considered. ${ }^{40} \mathrm{RBD}$ is recognised in $50-80 \%$ of patients with DLB. ${ }^{26}$ Disturbed arousal is thought to be a component of the fluctuations that occur in DLB, suggesting abnormalities in the normal sleep-wake cycle. Abnormalities in the sleep-wake cycle are a characteristic feature of delirium, ${ }^{8}$ and disturbances in consciousness are also common, with impairments of alertness, awareness and arousal. ${ }^{28}$

\section{Emotional disturbances}

Emotional disturbances are present in DLB and delirium. Depression is a common presenting feature of DLB however, in a population where apathy and psychomotor retardation are common, diagnosis may not always be clear-cut. ${ }^{26}$ Lability of affect occurs in delirium, and there are also studies which suggest more sustained mood disturbances in delirium, including depression, ${ }^{41}$ particularly in the hypoactive subtype.

The clinical similarities or otherwise between DLB and delirium are summarised in table 2.

\section{Pathophysiology}

$\alpha$-Synuclein

Lewy bodies are spherical intracytoplasmic inclusion bodies, composed of abnormally phosphorylated neurofilament proteins aggregated with ubiquitin and $\alpha$-synuclein, ${ }^{4}$ found in cortical and subcortical regions in DLB. ${ }^{42}$ Lewy body formation and neuronal loss in the brainstem nuclei, particularly the substantia nigra, leads to the movement disorder seen in DLB. ${ }^{4}$ $\alpha$-synuclein has not been linked with delirium.

\section{Dopamine}

DLB is associated with profound dopaminergic changes with marked nigrostriatal loss. ${ }^{37}$ A loss of dopamine receptors in the cerebral cortex in DLB may explain the profound sensitivity to neuroleptic medications (dopamine antagonists) manifesting as EPS, altered consciousness and increased confusion. ${ }^{22}$ Typical antipsychotics should therefore be avoided in DLB. ${ }^{43}$ There have been no postmortem studies investigating the dopaminergic system in delirium, however there does appear to be a link between the dopaminergic pathway and delirium, possibly a state of dopaminergic excess. ${ }^{41}$ Evidence for this comes from observations that dopaminergic medications, amphetamines and 
Table 2 Comparison of the clinical features of DLB and delirium

\begin{tabular}{|c|c|c|}
\hline DLB & Delirium & Similarity \\
\hline Gradual, progressive onset & Acute onset & No \\
\hline $\begin{array}{l}\text { Global cognitive impairment (particularly attention, working memory, } \\
\text { visuospatial, some executive functions) }\end{array}$ & $\begin{array}{l}\text { Cognitive impairment (particularly attention, orientation, memory, } \\
\text { visuospatial, vigilance) }\end{array}$ & Yes \\
\hline Fluctuating cognition & Fluctuating cognition & Yes \\
\hline Recurrent visual hallucinations & Visual hallucinations & Yes \\
\hline Spontaneous features of parkinsonism & Psychomotor disturbances & Some \\
\hline Repeated falls & Risk of falls & Some \\
\hline Syncope & Not reported & No \\
\hline Transient loss of consciousness & Fluctuating consciousness & Yes \\
\hline Neuroleptic sensitivity & Some symptoms responsive to neuroleptics for example, agitation, psychosis & No \\
\hline Delusions & Delusions & Yes \\
\hline Hallucinations in other modalities & Hallucinations in other modalities & Yes \\
\hline REM sleep behaviour disorder & Disturbances in the sleep-wake cycle & Some \\
\hline Emotional disturbances & Emotional disturbances & Yes \\
\hline
\end{tabular}

DLB, dementia with Lewy bodies; REM, rapid eye movement.

electroconvulsive therapy, all of which stimulate dopamine in some way, can result in delirium. ${ }^{44}$ Conversely dopamine antagonists, such as typical antipsychotic medications, are part of the pharmacological management of delirium. ${ }^{45}$

\section{Acetylcholine}

DLB is associated with extensive loss of brainstem and basal forebrain cholinergic projection neurons. ${ }^{4} \mathrm{~A}$ widespread reduction of choline acetyltransferase, the presynaptic enzyme for acetylcholine, occurs in the brains of those with DLB, and is thought to occur early in the disease process. ${ }^{46}$ Cholinesterase inhibitors have been shown to be of benefit for cognitive and neuropsychiatric symptoms of DLB. ${ }^{6}$

A state of cholinergic deficiency is one of the leading hypothetical mechanisms for delirium ${ }^{23}$ and elevated anticholinergic activity correlates positively with delirium severity. ${ }^{47}$ Anticholinergic medications and prior dementia, in which cerebral cholinergic deficiency is prominent, both predict delirium. ${ }^{48}$ Field et al looked at the interaction between acute systemic inflammation and chronic cholinergic hypofunction, and found that on a background of chronic cholinergic depletion, an acute systemic inflammatory insult resulted in acute cognitive deficits and delirium. ${ }^{48}$ This suggests a role for cholinergic loss and inflammation in the pathophysiology of delirium. This state of cholinergic deficiency may explain why patients with pre-existing dementia are at increased likelihood of developing delirium, since their brain is already in a state of cholinergic deficit. With additional insults (an acute inflammatory reaction secondary to infection, for example) a delirium is precipitated. The cholinergic deficiency hypothesis makes delirium a potential target for treatment with acetylcholinesterase inhibitors. ${ }^{44}$ Although early case reports on cholinesterase inhibitors for delirium showed some positive results, two double-blind, randomised trials ${ }^{49} 50$ failed to demonstrate statistically significant efficacy of cholinesterase inhibitors in the prevention and treatment of postoperative delirium. As such, cholinesterase inhibitors are not currently recommended for the prevention and management of delirium.

\section{Genetics}

The ApoE- $\varepsilon 4$ allele has been reported to be a risk factor for DLB. Previous neuropathological studies have demonstrated similar frequencies of the ApoE- $\mathbf{\varepsilon} 4$ allele in patients with a preexisting diagnosis of probable $\mathrm{AD}$ and probable DLB according to the National Institute of Neurological and Communicative Diseases and Stroke/Alzheimer's Disease and Related Disorders Association (NINCDS/ADRDA) criteria and DLB consensus criteria, respectively. ${ }^{51}$ More recently studies have explored the ApoE- $\varepsilon 4$ allele in relation to delirium, though the results have been conflicting. ${ }^{52}$ Some studies have shown an association between ApoE- $\boldsymbol{\varepsilon} 4$ allele and delirium, ${ }^{53}$ furthermore some have shown an association with duration of delirium. ${ }^{54}$ Yet other studies have shown no such association (see ${ }^{52}$ for references). The reason for such conflicting evidence may relate to the small samples of participants included in the published studies. ${ }^{52}{ }^{54} \mathrm{It}$ is also important to mention that many of the studies have not taken into account the important known association between the ApoE- $\varepsilon 4$ allele and pre-existing dementia. ${ }^{52}$

\section{Neuroimaging}

Structural imaging studies in DLB have consistently shown relative preservation of the medial temporal lobe and hippocampus in DLB when compared with $\mathrm{AD}^{55}$ (figure 1). Generalised cortical atrophy is also commonly seen, though the frontal, parieto-occipital and subcortical regions are most affected. ${ }^{55}$ White matter hyperintensities on MRI are more frequent in DLB cases than in controls, but occur to a similar extent as AD. ${ }^{56}$

Neuroimaging in delirium is much less researched. This may be because of the practical difficulties of scanning in the acutely ill elderly person with delirium. A systematic review carried out in 2008 found only 12 imaging studies with a total of 194 patients with delirium. ${ }^{57}$ Structural imaging studies showed that patients with delirium had increased brain atrophy, ventricular enlargement and increased white matter lesions, as well as basal ganglia changes. ${ }^{57}$ One study looked at older patients with depression who were being treated with $\mathrm{ECT}^{58}$; MRI scans were carried out prior to electroconvulsive therapy, and subjects who subsequently developed delirium had higher numbers of basal ganglia lesions than those who did not develop delirium. This apparent involvement of the basal ganglia in delirium could explain the overlap of a number of clinical features in delirium and DLB. In DLB, hypoperfusion on SPECT and hypometabolism on positron emission tomography imaging are found in the occipital and posterior parietal cortices. ${ }^{59} \mathrm{~A}$ number of studies have been conducted looking at cerebral perfusion in delirium. ${ }^{60}$ One study looking at cerebral perfusion using xenon CT found global hypoperfusion during the episode 


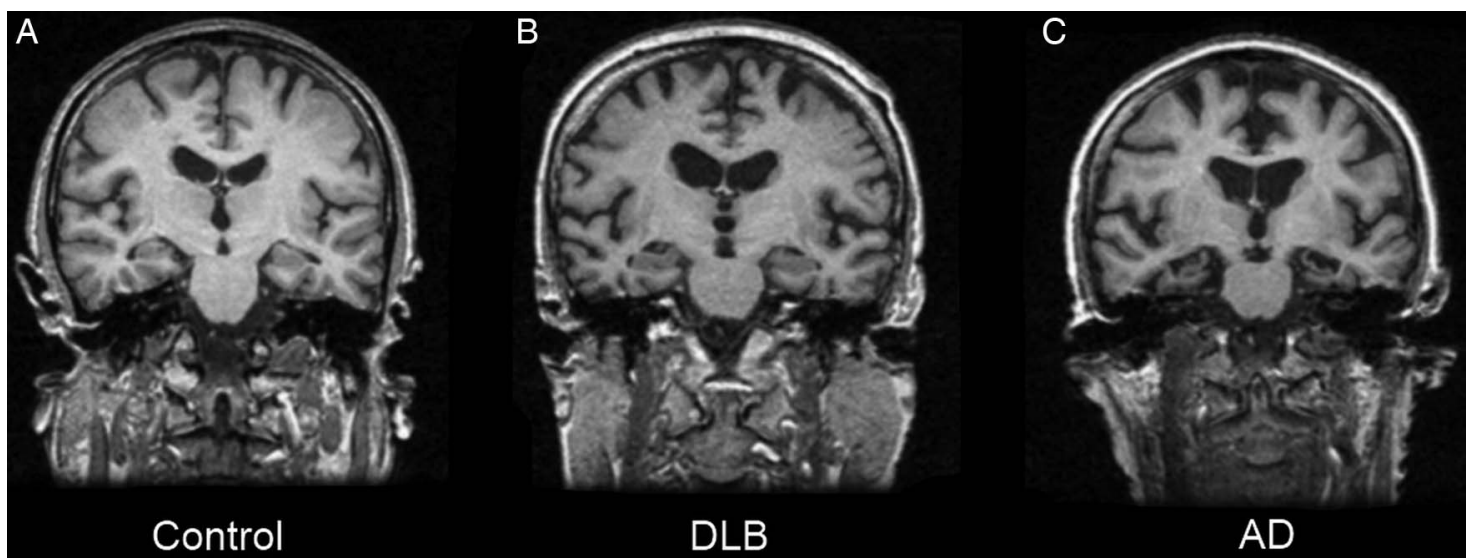

Figure 1 Coronal view of a structural MRI brain scan in (A) Control, (B) Dementia with Lewy bodies (DLB) and (C) AD. Note the relatively preserved medial temporal lobes in DLB compared with AD.

of delirium, particularly in subcortical structures and the occipital cortex. ${ }^{61}$ Another study found regional blood flow abnormalities in $50 \%$ of patients with delirium using hexamethylpropylene amine oxime SPECT scanning, ${ }^{62}$ with reduced blood flow in the frontal and parietal regions. An additional finding is that regional cerebral blood flow recovers after the episode of delirium is resolved, perhaps reflecting the acute nature of delirium. ${ }^{61}$ These findings suggest that DLB and delirium are both states of cerebral hypoperfusion.

The most clinically useful diagnostic imaging techniques in DLB is striatal dopaminergic imaging, using ${ }^{123} \mathrm{I}-\mathrm{N}$-omega-fluoropropyl-2-beta-carboxymethoxy-3beta-(4-iodophenyl)nortro-

pane $\left({ }^{123}\right.$ I-FP-CIT) SPECT (DaT Scanning). ${ }^{63}$ This has a sensitivity of $78 \%$, a specificity of $90 \%$ and an overall accuracy of $86 \%$ in diagnosing DLB. ${ }^{64}$ We are not aware of any studies in delirium using ${ }^{123}$ I-FP-CIT SPECT. ${ }^{57}$

\section{CSF biomarkers}

The current evidence regarding CSF and blood biomarkers in DLB and delirium is summarised in table 3.

\section{Acetylcholine}

Acetylcholinesterase (AChE) levels in CSF have been studied in delirium. ${ }^{65}$ Koponen et $a l^{65}$ found no association between CSF AChE levels and delirium, but did report a drop in AChE levels between 2 weeks and 1 year following an episode of delirium in patients with comorbid $\mathrm{AD}$ and multi-infarct dementia, $\mathrm{AD}$ or delirium and multi-infarct dementia. ${ }^{65}$ This suggests an association between a reduction in CSF AChE levels and dementia. Interestingly this reduction was not present in the subgroup of patients with delirium with Parkinson's Disease (PD), which shares some of the neuropathological features of DLB. 5-HIAA, a metabolite of dopamine, was found to be higher in subjects with delirium and $\mathrm{VaD}$ compared with controls, ${ }^{66}$ again suggesting a role for dopamine in delirium.

\section{$\alpha$-Synuclein}

Quantifying levels of $\alpha$-synuclein in CSF has received a great deal of attention in neurodegenerative disorders, although the results from studies conducted thus far appear conflicting. Mollenhauer et $a l^{67}$ compared DLB and PD with AD and control subjects and found significantly lower levels of CSF $\alpha$-synuclein DLB and PD. Kasuga et $a l^{68}$ compared DLB subjects with $\mathrm{AD}$, and other dementias; in this study levels of CSF $\alpha$-synuclein were significantly lower in the DLB subjects compared with AD and the other dementias studied. Conversely Noguchi-Shinohara et al ${ }^{69}$ found that levels of CSF $\alpha$-synuclein do not differ between DLB and $\mathrm{AD}$, however they did find that lower levels of CSF $\alpha$-synuclein correlated with a longer DLB duration, perhaps reflecting a more advanced stage of disease. Spies $e t a l^{70}$ did not find any significant differences in CSF $\alpha$-synuclein levels between DLB subjects and $\mathrm{AD}, \mathrm{VaD}$ and frontotemporal lobe dementia. Another study found a significant reduction in CSF $\alpha$-synuclein levels in patients with mild DLB, ${ }^{71}$ suggesting a role for CSF $\alpha$-synuclein as a biomarker for early DLB. $\alpha$-Synuclein as a biomarker has not been studied in delirium.

Tau

Tau has been investigated as a potential biomarker for DLB. ${ }^{72}$ Several studies report that CSF concentrations of tau protein are significantly lower in DLB than $\mathrm{AD},{ }^{46}{ }^{72}$ suggesting a role for CSF tau measurement as a biomarker to differentiate between these two diagnoses, rather than as a diagnostic tool for DLB. It also reflects a difference in the pathophysiology of DLB and $\mathrm{AD}$. In one study looking at CSF tau levels in postoperative delirium, there was no significant association between levels of CSF tau and delirium in older adults hospitalised for surgical repair of a hip fracture. ${ }^{73}$

\section{Amyloid}

Abnormal processing of amyloid precursor protein leads to an accumulation of extracellular amyloid in brain tissue and a subsequent reduction in peripheral $\beta$ amyloid, measurable in CSF. Decreased levels of CSF $\beta$-amyloid have been reported for patients with AD and DLB compared with controls. ${ }^{74}$ A novel peptide called $\beta$-amyloid peptide thought to represent an interaction between $\beta$-amyloid and $\alpha$-synuclein has been measured in the CSF of patients with DLB, as well as AD. ${ }^{74}$ Levels of $\beta$-amyloid peptide were significantly increased in the CSF of DLB subjects compared with controls without dementia.

\section{Inflammatory biomarkers}

Inflammation is thought to be associated with amyloid accumulation, suggesting a role for inflammatory processes in DLB. ${ }^{72}$ There is also a greater prevalence of delirium in systemic inflammatory states. ${ }^{75}$ Inflammatory markers can be detected in CSF. ${ }^{72}$ In a study looking at CSF inflammatory markers in DLB and $\mathrm{AD}$, interleukin (IL) CSF levels were slightly but not significantly more elevated in DLB and AD subjects compared with controls. ${ }^{76}$ In delirium it is the role of cytokines as markers of 
Table 3 Comparative summary of the evidence for CSF and blood biomarkers in DLB and delirium ${ }^{34-52}$

\begin{tabular}{|c|c|c|c|}
\hline Biomarker & Fluid & Evidence in DLB & Evidence in delirium \\
\hline \multirow[t]{7}{*}{ Acetylcholine } & CSF & Not studied & No association ${ }^{65}$ \\
\hline & & & Some association with delirium $+\mathrm{AD} / \mathrm{VaD} \mathrm{D}^{65}$ \\
\hline & Blood & Not studied & $\uparrow S A A$ in delirium versus controls ${ }^{47}$ \\
\hline & & & $\uparrow S A A$ in greater number of delirium symptoms ${ }^{47}$ \\
\hline & & & $\uparrow S A A$ in acute delirium ${ }^{78}$ \\
\hline & & & \SAA following resolution of delirium ${ }^{78}$ \\
\hline & & & $\begin{array}{l}\Leftrightarrow \text { SAA in delirium versus controls and delirium } \pm \text { dementia }^{79} \\
\downarrow A C h E / B u C h E \text { in delirium } \\
80\end{array}$ \\
\hline \multirow{5}{*}{$\alpha$-synuclein } & CSF & $\alpha \alpha$-syn in DIB versus $A D /$ control 6768 & Not studied \\
\hline & & $\Leftrightarrow \alpha$-syn in DLB versus $A D_{1}^{69} V_{a D}, F_{L L D}{ }^{70}$ & INot studied \\
\hline & & $\downarrow \alpha-$ syn in mild DLB ${ }^{71}$ & \\
\hline & & $\downarrow \alpha$-syn in longer DLB duration ${ }^{69}$ & \\
\hline & Blood & $\downarrow \alpha-$ syn in DLB versus controls ${ }^{82}$ & Not studied \\
\hline Amyloid & CSF & $\begin{array}{l}\downarrow \beta \text {-amyloid in DLB versus controls }{ }^{74} \\
\uparrow A b \text {-ox in DLB versus controls }\end{array}$ & Not studied \\
\hline Tau & CSF & $\downarrow T a u$ in DLB versus $A D^{46} 72$ & No association with delirium ${ }^{73}$ \\
\hline \multirow[t]{6}{*}{ Inflammatory markers } & CSF & $\uparrow I L$ in DLB versus controls (not significant) ${ }^{76}$ & $\uparrow I L-6$ and IL-8 in delirium versus controls ${ }^{77}$ \\
\hline & Blood & $\uparrow T N F-\alpha$ associated with neuropsychiatric symptoms in mild/moderate DLB ${ }^{87}$ & 个IL- 6 and IL-8 in delirium versus controls ${ }^{84} 85$ \\
\hline & & 个IL-6 associated with poor cognitive function at baseline ${ }^{87}$ & $\uparrow$ IL-6 during delirium ${ }^{85}$ \\
\hline & & & १IL-8 before delirium ${ }^{85}$ \\
\hline & & & 个IL-6 in hyper versus hypoactive delirium ${ }^{85}$ \\
\hline & & & $\Leftrightarrow \mathbb{I L}$ in delirium ${ }^{\circ}$ \\
\hline
\end{tabular}

inflammation which has been most extensively studied. ${ }^{75}$ MacLullich $e t a l^{77}$ looked at cytokines IL-6 and IL-8 in the CSF of patients with delirium following hip fracture surgery; CSF IL-8 levels were significantly higher in delirium cases compared with controls and CSF IL-6 levels were also higher in the delirium group. Dementia was not associated with such increased levels.

\section{Blood biomarkers \\ Acetylcholine}

Serum anticholinergic activity (SAA) has been studied in delirium. Flacker $e t a l^{47}$ found SAA levels to be more than doubled in delirium, and increased levels of SAA were also associated with a higher burden of delirium symptoms. Further work by the same group found that SAA levels were significantly higher during acute illness than at 1-month follow-up, with a decline in SAA levels with the resolution of delirium. ${ }^{78}$ These findings were not replicated by Thomas et $a l^{79}$ who found no significant difference in SAA levels between subjects with dementia and additional delirium, subjects with dementia without delirium and cognitively unimpaired subjects. One hypothesis for this variation is that as SAA reflects peripheral anticholinergic activity only, it is not a true reflection of the central anticholinergic deficiency seen in delirium. ${ }^{75}$ Blood levels of AChE and butylcholinesterase have been shown to be decreased in people who develop a delirium. ${ }^{80}$ Cerejeira et $a l^{81}$ reported that in older patients undergoing elective hip surgery, preoperatively plasma esterase activity was significantly lower in patients who developed delirium compared with the remaining subjects. This suggests a predictive value for AChE.

\section{$\alpha$-Synuclein}

There is evidence that levels of $\alpha$-synuclein are altered in blood in DLB subjects. ${ }^{82}$ Laske et al found significantly reduced $\alpha$-synuclein serum levels in DLB compared with AD and control subjects. Foulds et al have developed immunoassays to detect levels of total and oligomeric forms of $\alpha$-synuclein, and phosphorylated and phosphorylated oligomeric forms of $\alpha$-synuclein within biological samples such as CSF, with a view of identifying a biomarker for the synucleinopathies. ${ }^{83}$

\section{Inflammatory biomarkers}

Measurement of serum levels of cytokines as biomarkers for delirium have been studied. ${ }^{75}$ One study of medical inpatients found significantly higher IL-6 levels and IL-8 levels in patients with delirium compared with those without. ${ }^{84}$ Van Munster et $a l^{85}$ also detected elevated levels of serum IL-6 and IL-8 in delirium, with IL-8 levels highest prior to the onset of delirium, and levels of IL- 6 highest during the delirium. Patients with hyperactive delirium also had higher IL-6 levels than patients with hypoactive delirium, suggesting specifically that the former subtype may be the result of an inflammatory mediated response. Other studies do not replicate these results. ${ }^{86}$

Blood inflammatory biomarkers have been recently studied in DLB. ${ }^{87}$ Clough et al looked at serum IL-6 and tumour necrosis factor $\alpha$ levels in 35 community-dwelling subjects with mild or moderate DLB. In these subjects, raised levels of TNF- $\alpha$ were associated with a greater burden of neuropsychiatric symptoms, without any evidence of an associated delirium or any systemic illness. This suggests that systemic inflammation may be related to the development of neuropsychiatric symptoms in DLB. This is similar to findings in $\mathrm{AD}$, where elevated proinflammatory cytokine is also associated with greater neuropsychiatric symptom burden. ${ }^{88}$ Increased levels of IL- 6 were also associated with poorer baseline cognitive function. This was, however, a small cross-sectional study and further work in this area is needed, with such work also examining for the potential significance of intercurrent infections, which may exacerbate symptoms and even subsequently decline, possibly though inflammatory mechanisms.

\section{Delirium or prodromal DLB?}

It is established that delirium is independently associated with cognitive decline and dementia. ${ }^{17} 18$ There is also evidence that 
delirium is more common in the very early stages of $\mathrm{DLB}^{89-91}$ suggesting that delirium may be more of a prodrome of DLB, rather than simply a risk factor for dementia per se. Of note the available evidence relies on studies with small numbers of subjects, and replication with larger numbers would be welcomed.

Rognve et $a l^{89}$ carried out a small study in western Norway to explore the presenting and early features of patients with DLB. It was found that prior to diagnosis, almost half (43\%) of those with DLB in the study had symptoms suggestive of delirium. Visual hallucinations, falling and psychomotor disturbances were also reported prediagnosis, all of which could represent an undiagnosed delirium. Gaig et $a l^{90}$ studied six cases of rapidly progressive DLB and found that half had an episode of delirium at the onset of their disease. In two-thirds of patients, slight cognitive and behavioural symptoms were present several months before the appearance of the delirium.

One small study of pathologically confirmed cases of dementia suggested an episode of delirium to be more common in the predementia phase of DLB as compared with AD. ${ }^{91}$ This study was concerned with the mild cognitive impairment (MCI) stage of DLB and compared this with the MCI stage of AD, and showed that those with MCI-DLB had more episodes of delirium in the preclinical disease state of DLB compared with the MCI-AD cases. This led to the conclusion that such features may lead to early detection of prodromal DLB.

Of course, not all delirium will progress to DLB, some will not progress and others will be a prodrome of other dementias, like Alzheimer's disease. What requires further study is whether there may be different manifestations of delirium, or specific clinical features, which may predict which cases are more likely to progress to DLB than others.

\section{Delirium outcomes}

A number of studies have looked at the outcomes of delirium over time. Rockwood et al studied the risk of dementia and death after delirium in 38 patients with delirium and 148 patients without delirium. ${ }^{18}$ The incidence of dementia after an episode of delirium was $18.1 \%$ per year over 3 years, compared with $5.6 \%$ in patients without delirium, with an unadjusted relative risk of dementia of 3.23 in the delirium group.

A study carried out in Sweden investigated whether delirium in older patients with femoral neck fractures was associated with developing dementia, and found that $30 / 78$ cases $(38.5 \%)$ developed dementia within the follow-up time of 5 years. ${ }^{92}$ Of these, two-thirds had suffered from an episode of delirium postoperatively, indicating that delirium increases the likelihood of dementia. The dementia subtype was not indicated. Another study looked at patients with hip surgery who were participating in a clinical trial of haloperidol prophylaxis for delirium and followed them up for 30 months. ${ }^{93}$ During follow-up $54.9 \%$ of the patients with delirium had died versus $34.1 \%$ of the controls. Of the surviving patients, $77.8 \%$ of the patients with postoperative delirium developed dementia or MCI versus $40.9 \%$ of the control patients (Relative risk (RR) 1.9). This suggests that postoperative delirium could be a marker of undetected dementia, and also highlights the high mortality rate following an episode of delirium. What is interesting to consider is the possibility that some of the $54.9 \%$ of patients who died following this study may in fact have had early DLB, been medicated with haloperidol for the purpose of the clinical trial, and whether neuroleptic sensitivity may have played a role in their outcome. This is clearly speculative, however it highlights the challenges clinicians are faced with when dealing with these diagnoses.
A meta-analysis by Witlox et al looked at 51 articles with a total of 714 patients with delirium and 2243 controls. ${ }^{16}$ Two studies which included 241 participants (56 delirium, 185 controls) looked at the risk of dementia after delirium, and the pooled results showed that the patients in the delirium group had an increased risk of dementia compared with controls, $(62.5 \%$ vs $8.1 \%$, OR 12.52 , after 3.2 years and 5.0 years of follow-up). ${ }^{16}$

In a recent study, ${ }^{19}$ Davis et al carried out a population-based cohort study looking at delirium outcomes. The group found that a single episode of delirium increases the risk of dementia (OR 8.7, 95\% CI 2.1 to 35 ) and that delirium is also associated with worsening dementia severity in subjects with a pre-existing diagnosis (OR 3.1, 95\% CI 1.5 to 6.3 ).

This is all very strong evidence that delirium significantly increases the risk of developing cognitive impairment and dementia, increases the risk of death and worsens pre-existing dementia. There is some evidence to suggest that delirium accelerates cognitive decline in Alzheimer's disease, ${ }^{94}$ suggesting a potential link between delirium and $\mathrm{AD}$, or perhaps dementia in general. Further work looking at dementia subtype post delirium, particularly DLB, would be welcome given the findings presented in this review.

\section{DISCUSSION}

There are a number of clinical features which are shared by DLB and delirium which can make differentiating these diagnoses difficult, particularly at the initial stages of presentation. The possibility of an underlying diagnosis of DLB should be considered in a patient presenting with what appears to be delirium. As such, the management of delirium is also an important issue and something that should be optimised at the time of symptoms. A full discussion surrounding this is beyond the scope of this review, however one must consider the potential for neuroleptic sensitivity, and caution needs to be taken when using typical antipsychotics in the management of what is thought to be delirium. Certainly in patients with additional features of DLB, such medications should be avoided given the potential for serious adverse reactions. ${ }^{43}$ There is an argument for services to be developed to allow patients presenting with delirium to be followed up and monitored for emerging dementia, potentially leading to early diagnosis of dementia if detected.

Cholinergic deficiency appears to play a key role in the pathophysiology of DLB and delirium, suggesting a common aetiological mechanism. Increasing our understanding of alterations in the cholinergic system in DLB and delirium could aid our understanding of the links between these diagnoses further. The role of the dopaminergic system in DLB has been well established, however further work is needed to look into the dopaminergic system in delirium, as the evidence presented thus far is somewhat incidental. There are several areas where data are lacking which limit the ability to draw conclusions between delirium and DLB, most notably in pathological and imaging studies of amyloid and synuclein changes in delirium that would have clear relevance to DLB and other types of dementia.

Some of the neuroimaging studies reviewed suggest features of brain changes which are common to DLB and delirium, such as basal ganglia pathology. The basal ganglia pathology in delirium was predominately of vascular aetiology and may simply represent a brain vulnerability which may then lead to an increased risk of developing dementia in the future. The practical challenges surrounding scanning an elderly population with delirium may unfortunately limit future research in this very interesting and important area. 
We have shown that delirium is a risk factor for dementia and cognitive impairment, with rates of dementia being significantly increased compared with the baseline rate and this suggests a common pathway in these two diagnoses. Since delirium and dementia are both age-related conditions, in future studies it would be interesting to stratify the age of appearance of dementia after the episode of delirium, in order to see if age at onset, and duration between the episode of delirium and the onset dementia are related.

There is little published data thus far regarding whether any particular type of dementia is associated with delirium, and although the evidence presented in this review suggests that there are certainly links between delirium and DLB, there is evidence to suggest that other dementia subtypes have links with delirium. ${ }^{20}{ }^{21}$ It may be that there is no single aetiological mechanism for the heterogenous condition that is delirium, so perhaps some, but not all delirium will predispose to, or be part of a prodrome of dementia. This could be addressed by stratifying delirium by phenomenological or aetiological subtype in future studies and subsequent diagnosis of a dementia by subtype. One might hypothesise that particular features, if very prominent, for example visual hallucinations, sleep disorder, motor features and fluctuations, may make cases more likely to be prodromal DLB, but evidence is lacking and this needs to be tested in prospective studies.

We have reviewed the role of CSF and serum biomarkers to detect DLB and delirium. So far the evidence for a single biomarker to detect either condition is conflicting; however this is an important area for future research. The role of inflammation in both diagnoses is an interesting area which is supported by the evidence discussed surrounding inflammatory biomarkers. Randomised controlled trials looking at the role of antiinflammatory agents in both diagnoses could be beneficial.

If biomarkers for DLB could be detected at the time of an episode of delirium, these may then predict those patients who are either showing signs of very early DLB, or those who are going to develop DLB in the future. Potential biomarkers of interest in longitudinal studies of people with delirium would include polysomnography (for RBD and other sleep disorders), FP-CIT imaging to detect early dopaminergic transporter loss, autonomic function tests (cardiac Metaiodobenzylguanidine 5-HIAA: 5-Hydroxyindoleacetic acid 123I-FP-CIT: (123)I-Nomega-fluoropropyl-2-beta-carboxymethoxy-3beta-(4-iodophenyl) nortropane (MIBG) imaging) to detect early dysautonomia and cardiac denervation. It may also be that delirium itself can serve as a 'marker' for dementia and represent a diagnostic opportunity for dementia. This could lead to earlier diagnosis of dementia and offer target for early treatment. Further research is clearly needed to challenge this novel hypothesis.

\author{
Author affiliations \\ ${ }^{1}$ Institute for Ageing and Health, Newcastle University, Campus for Ageing and \\ Vitality, Newcastle upon Tyne, UK \\ ${ }^{2}$ Department of Old Age Psychiatry, Northumberland Tyne and Wear NHS Trust, \\ Morpeth, Northumberland, UK \\ ${ }^{3}$ Department of Older Peoples Medicine, Newcastle upon Tyne Hospitals NHS \\ Foundation Trust, Freeman Hospital, Newcastle upon Tyne, UK \\ ${ }^{4}$ Department of Psychiatry, University of Cambridge, Cambridgeshire and \\ Peterborough NHS Foundation Trust, Level E4 Cambridge Biomedical Campus, \\ Cambridge, UK
}

Acknowledgements This work was supported by the UK NIHR Biomedical Research Centre and Biomedical Research Unit in Lewy body dementia awarded to the Newcastle upon Tyne Hospitals NHS Foundation Trust and the NIHR Biomedical Research Centre and Biomedical Research Unit in Dementia awarded to Cambridge University Hospitals NHS Trust and the University of Cambridge.
Contributors RLG undertook the literature search, wrote and organised the manuscript. ERLCV provided supervision for the project, summarised the current data on genetics, read, reviewed and made amendments to all sections of the manuscript. JTOB provided supervision for the project, read, reviewed and made amendments to all sections of the manuscript. All authors approved the final manuscript for submission.

Funding At the time of writing Dr Rachel Gore was an Academic Clinical Fellow in Psychiatry funded partly by the National Institute of Health Research (NIHR).

Competing interests None.

Provenance and peer review Not commissioned; externally peer reviewed.

\section{REFERENCES}

1 Knapp M, Prince E, Albanese E. A report into the prevalence and cost of dementia'. London, UK: Alzheimer's Society, 2007.

2 Kosaka K, Yoshimura M, Ikeda K, et al. Diffuse type of Lewy body disease: progressive dementia with abundant cortical Lewy bodies and senile changes of varying degree-a new disease? Clin Neuropathol 1984;3:185-92.

3 Singh D. Diagnosis of dementia with Lewy bodies in clinical practice: a literature review. Australas Psychiatry 2005;13:54-9.

4 McKeith IG. Dementia with Lewy bodies. Br J Psychiatry 2002;180:144-7.

5 Vann Jones SA, O'Brien JT. The prevalence and incidence of dementia with Lewy bodies: a systematic review of population and clinical studies. Psychol Med 2014:44:673-83.

6 McKeith IG, Dickson DW, Lowe J, et al. Diagnosis and management of dementia with Lewy bodies: third report of the DLB Consortium. Neurology 2005;65:1863-72.

7 McKeith IG, Ballard CG, Perry RH, et al. Prospective validation of consensus criteria for the diagnosis of dementia with Lewy bodies. Neurology 2000;54:1050-8.

8 Gupta N, de Jonghe Jos, Schieveld Jan, et al. Delirium phenomenology: what can we learn from the symptoms of delirium? J Psychosom Res 2008;65:215-22.

9 de Lange E, Verhaak PF, van der Meer K. Prevalence, presentation and prognosis of delirium in older people in the population, at home and in long term care: a review. Int J Geriatr Psychiatry 2013;28:127-34.

10 Siddiqi $\mathrm{N}$, House AO, Holmes JD. Occurrence and outcome of delirium in medical in-patients: a systematic literature review. Age Ageing 2006;35:350-64.

11 APA, American Psychiatric Association. Diagnostic and statistical manual of mental health disorders. 5th edn. Washington, DC: American Psychiatric Publishing, 2013.

12 Meagher D. Motor subtypes of delirium: past, present and future. Int Rev Psychiatry 2009;21:59-73.

13 Inouye SK, Inouye SK, van Dyck $\mathrm{CH}$, et al. Clarifying confusion: the confusion assessment method. A new method for detection of delirium. Ann Intern Med 1990;113:941-8.

14 WHO, World Health Organisation. ICD-10 Classifications of Mental and Behavioural Disorder: Clinical Descriptions and Diagnostic Guidelines, Geneva, 1992

15 Francis J, Martin D, Kapoor WN. A prospective study of delirium in hospitalized elderly. JAMA 1990;263:1097-101.

16 Witlox J, Eurelings LSM, de Jonghe JFM, et al. Delirium in elderly patients and the risk of postdischarge mortality, institutionalization, and dementia: a meta-analysis. JAMA 2010;304:443-51.

17 MacLullich AMJ, Beaglehole A, Hall RJ, et al. Delirium and long-term cognitive impairment. Int Rev Psychiatry 2009;21:30-42.

18 Rockwood K, Cosway S, Carver D, et al. The risk of dementia and death after delirium. Age Ageing 1999:28:551-6.

19 Davis DH, Muniz Terrera $\mathrm{G}$, Keage $\mathrm{H}$, et al. Delirium is a strong risk factor for dementia in the oldest-old: a population-based cohort study. Brain 2012;135(Pt 9):2809-16.

20 Rahkonen T, Rahkonen T, Luukkainen-Markkula R, et al. Delirium episode as a sign of undetected dementia among community dwelling elderly subjects: a 2 year follow up study. J Neurol Neurosurg Psychiatry 2000;69:519-21.

21 Rahkonen T, Rahkonen T, Eloniemi-Sulkava U, et al. Delirium in the non-demented oldest old in the general population: risk factors and prognosis. Int J Geriatr Psychiatry 2001;16:415-21.

22 Gold G. Dementia with Lewy bodies: clinical diagnosis and therapeutic approach. Front Neurol Neurosci 2009;24:107-13.

23 Inouye SK. Delirium in older persons. N Engl J Med 2006;354:1157-65.

24 Hales R. Essentials of Psychiatry. 3rd edn. American Psychiatric Publishing, Inc, 2010.

25 Dasgupta M, Hillier LM. Factors associated with prolonged delirium: a systematic review. Int Psychogeriatr 2010;22:373-94.

26 McKeith I. Dementia with Lewy Bodies: a clinical and historical overview. In Ames D, Burns A, O'Brien J.eds Dementia. Great Britain: Edward Arnold, 2010:597-607.

27 Simard M, van Reekum $\mathrm{R}$, Cohen $\mathrm{T}$. A review of the cognitive and behavioral symptoms in dementia with Lewy bodies. J Neuropsychiatry Clin Neurosci 2000;12:425-50. 
28 Cerejeira J, Mukaetova-Ladinska EB. A clinical update on delirium: from early recognition to effective management. Nurs Res Pract 2011;2011:875196.

29 Meagher DJ, Moran M, Raju B, et al. Phenomenology of delirium. Assessment of 100 adult cases using standardised measures. Br J Psychiatry 2007;190: 135-41.

30 Brown LJ, McGrory S, McLaren L, et al. Cognitive visual perceptual deficits in patients with delirium. J Neurol Neurosurg Psychiatry 2009;80:594-9.

31 Walker MP, Ayre GA, Cummings JL, et al. Quantifying fluctuation in dementia with Lewy bodies, Alzheimer's disease, and vascular dementia. Neurology 2000;54:1616-25.

32 Walker MP, Ayre GA, Cummings JL, et al. The clinician assessment of fluctuation and the one day fluctuation assessment scale. Two methods to assess fluctuating confusion in dementia. Br J Psychiatry 2000;177:252-6.

33 Bhat R, Rockwood K. Delirium as a disorder of consciousness. J Neurol Neurosurg Psychiatry 2007;78:1167-70.

34 Neef $D$, Walling AD. Dementia with Lewy bodies: an emerging disease. Am Fam Physician 2006:73:1223-9.

35 McKeith I. Dementia with Lewy bodies. Dialogues Clin Neurosci 2004;6:333-41.

36 Lipowski ZJ. Transient cognitive disorders (delirium, acute confusional states) in the elderly. Am J Psychiatry 1983;140:1426-36.

37 Mosimann UP, McKeith IG. Dementia with Lewy bodies-diagnosis and treatment. Swiss Med Weekly 2003;133:131-42.

38 Lakatos BE, Capasso V, Mitchell MT, et al. (Falls in the general hospital: association with delirium, advanced age, and specific surgical procedures. Psychosomatics 2009;50:218-26

39 Hartikainen $S$, Lonnroos E, Louhivuori K. Medication as a risk factor for falls: critical systematic review. J Gerontol A Biol Sci Med Sci 2007;62:1172-81.

40 Boeve BF, Saper CB. REM sleep behavior disorder: a possible early marker for synucleinopathies. Neurology 2006;66:796-7.

41 Leonard M, Spiller J, Keen J, et al. Symptoms of depression and delirium assessed serially in palliative-care inpatients. Psychosomatics 2009;50:506-14.

42 Ince $\mathrm{P}$, Halliday G. Pathology of dementia with Lewy bodies. In Ames D, Burns A O'Brien J, eds. Dementia. Great Britain: Edward Arnold, 2010:608-18.

43 McKeith I, Fairbairn A, Perry $R$, et al. Neuroleptic sensitivity in patients with senile dementia of Lewy body type. BMJ 1992;305:673-8.

44 Hshieh TT, Fong TG, Marcantonio ER, et al. Cholinergic deficiency hypothesis in delirium: a synthesis of current evidence. J Gerontol A Biol Sci Med Sci 2008;63:764-72

45 Platt MM, Breitbart W, Smith $M$, et al. Efficacy of neuroleptics for hypoactive delirium. J Neuropsychiatry Clin Neurosci 1994;6:66-7.

46 Sinha N, Firbank M, O'Brien JT. Biomarkers in dementia with Lewy bodies: a review. Int J Geriatr Psychiatry 2012;27:443-53.

47 Flacker JM, Cummings V, Mach JR, et al. The association of serum anticholinergic activity with delirium in elderly medical patients. Am J Geriatr Psychiatry 1998;6:31-41.

48 Field RH, Gossen A, Cunningham C. Prior pathology in the basal forebrain cholinergic system predisposes to inflammation-induced working memory deficits: reconciling inflammatory and cholinergic hypotheses of delirium. J Neurosci 2012;32:6288-94.

49 Liptzin B, Laki A, Garb JL, et al. Donepezil in the prevention and treatment of post-surgical delirium. Am J Geriatr Psychiatry 2005;13:1100-6.

50 Sampson EL, Raven PR, Ndhlovu PN, et al. A randomized, double-blind, placebo-controlled trial of donepezil hydrochloride (Aricept) for reducing the incidence of postoperative delirium after elective total hip replacement. Int J Geriatr Psychiatry 2007;22:343-9.

51 Kobayashi S, Tateno M, Park TW, et al. Apolipoprotein E4 frequencies in a Japanese population with Alzheimer's disease and dementia with Lewy bodies. PLOS ONE 2011;6:e18569.

52 van Munster BC, de Rooij SE, Korevaar JC. The role of genetics in delirium in the elderly patient. Dement Geriatr Cogn Disord 2009;28:187-95.

53 Leung JM, Sands LP, Wang Y, et al. Apolipoprotein E e4 allele increases the risk of early postoperative delirium in older patients undergoing noncardiac surgery. Anesthesiology 2007;107:406-11.

54 Ely EW, Girard TD, Shintani AK, et al. Apolipoprotein E4 polymorphism as a genetic predisposition to delirium in critically ill patients. Crit Care Med 2007;35:112-17

55 Watson R, Blamire AM, O'Brien JT. Magnetic resonance imaging in lewy body dementias. Dement Geriatr Cogn Disord 2009;28:493-506.

56 Barber R, Scheltens P, Gholkar A, et al. White matter lesions on magnetic resonance imaging in dementia with Lewy bodies, Alzheimer's disease, vascular dementia, and normal aging. J Neurol Neurosurg Psychiatry 1999;67:66-72.

57 Soiza RL, Sharma V, Ferguson K, et al. Neuroimaging studies of delirium: a systematic review. J Psychosom Res 2008;65:239-48.

58 Figiel GS, Coffey CE, Djang WT, et al. Brain magnetic resonance imaging findings in ECT-induced delirium. J Neuropsychiatry Clin Neurosci 1990;2:53-8.

59 O'Brien JT. Role of imaging techniques in the diagnosis of dementia. Br J Radiol 2007;80(Spec No 2):S71-7.
60 Alsop DC, Fearing MA, Johnson $\mathrm{K}$, et al. The role of neuroimaging in elucidating delirium pathophysiology. J Gerontol A Biol Sci Med Sci 2006;61:1287-93.

61 Yokota H, Ogawa S, Kurokawa A, et al. Regional cerebral blood flow in delirium patients. Psychiatry Clin Neurosci 2003;57:337-9.

62 Fong TG, Bogardus ST, Daftary A, et al. Cerebral perfusion changes in older delirious patients using 99mTc HMPAO SPECT. J Gerontol A Biol Sci Med Sci 2006;61:1294-9.

63 O'Brien JT, McKeith IG, Walker Z, et al. Diagnostic accuracy of 123I-FP-CIT SPECT in possible dementia with Lewy bodies. Br J Psychiatry 2009;194:34-9.

64 McKeith I, O'Brien J, Walker Z, et al. Sensitivity and specificity of dopamine transporter imaging with 123I-FP-CIT SPECT in dementia with Lewy bodies: a phase III, multicentre study. Lancet Neurol 2007:6:305-13.

65 Koponen HJ, Sirviö J, Lepola U, et al. A long-term follow-up study of cerebrospinal fluid acetylcholinesterase in delirium. Eur Arch Psychiatry Clin Neurosci 1994;243:347-51.

66 Koponen HJ, Lepola U, Leinonen E. A long-term follow-up study of cerebrospinal fluid 5-hydroxyindoleacetic acid in delirium. Eur Arch Psychiatry Clin Neurosci 1994;244:131-4

67 Mollenhauer B, Cullen V, Kahn I, et al. Direct quantification of CSF alpha-synuclein by ELISA and first cross-sectional study in patients with neurodegeneration. Exp Neurol 2008;213:315-25.

68 Kasuga K, Tokutake T, Ishikawa A, et al. Differential levels of alpha-synuclein, beta-amyloid42 and tau in CSF between patients with dementia with Lewy bodies and Alzheimer's disease. J Neurol Neurosurg Psychiatry 2010;81:608-10.

69 Noguchi-Shinohara M, Tokuda T, Yoshita M, et al. CSF alpha-synuclein levels in dementia with Lewy bodies and Alzheimer's disease. Brain Res 2009;1251: $1-6$.

70 Spies PE, Melis RJ, Sjögren MJ, et al. Cerebrospinal fluid $\alpha$-synuclein does not discriminate between dementia disorders. J Alzheimers Dis 2009;16:363-9.

71 Ballard C, Jones EL, Londos E, et al. alpha-Synuclein antibodies recognize a protein present at lower levels in the CSF of patients with dementia with Lewy bodies. Int Psychogeriatr 2010;22:321-7.

72 Mukaetova-Ladinska EB, Monteith R, Perry EK. Cerebrospinal fluid biomarkers for dementia with lewy bodies. Int J Alzheimers Dis 2010;2010:536538.

73 Witlox J, Kalisvaart KJ, de Jonghe JFM, et al. Cerebrospinal fluid beta-amyloid and tau are not associated with risk of delirium: a prospective cohort study in older adults with hip fracture. J Am Geriatr Soc 2011;59:1260-7.

74 Bibl M, Mollenhauer B, Esselmann $\mathrm{H}$, et al. CSF amyloid-beta-peptides in Alzheimer's disease, dementia with Lewy bodies and Parkinson's disease dementia. Brain 2006;129(Pt 5):1177-87.

75 Khan BA, Zawahiri M, Campbell NL, et al. Biomarkers for delirium-a review. J Am Geriatr Soc 2011;59(Suppl 2):S256-61.

76 Gomez-Tortosa E, Gonzalo I, Fanjul S, et al. Cerebrospinal fluid markers in dementia with lewy bodies compared with Alzheimer disease. Arch Neurol 2003;60:1218-22

77 MacLullich AM, Edelshain BT, Hall RJ, et al. Cerebrospinal fluid interleukin-8 levels are higher in people with hip fracture with perioperative delirium than in controls. J Am Geriatr Soc 2011;59:1151-3.

78 Flacker JM, Lipsitz LA. Serum anticholinergic activity changes with acute illness in elderly medical patients. J Gerontol A Biol Sci Med Sci 1999;54:M12-16.

79 Thomas C, Hestermann U, Kopitz J, et al. Serum anticholinergic activity and cerebral cholinergic dysfunction: an EEG study in frail elderly with and without delirium. BMC Neurosci 2008:9:86.

80 White S, Calver BL, Newsway V, et al. Enzymes of drug metabolism during delirium. Age Ageing 2005;34:603-8.

81 Cerejeira J, Batista P, Nogueira V, et al. Low preoperative plasma cholinesterase activity as a risk marker of postoperative delirium in elderly patients. Age Ageing 2011;40:621-6.

82 Laske C, Fallgatter AJ, Stransky E, et al. Decreased alpha-synuclein serum levels in patients with Lewy body dementia compared to Alzheimer's disease patients and control subjects. Dement Geriatr Cogn Disord 2011;31:413-16.

83 Foulds PG, Yokota O, Thurston A, et al. Post mortem cerebrospinal fluid alpha-synuclein levels are raised in multiple system atrophy and distinguish this from the other alpha-synucleinopathies, Parkinson's disease and Dementia with Lewy bodies. Neurobiol Dis 2012;45:188-95.

84 de Rooij SE, van Munster BC, Korevaar JC, et al. Cytokines and acute phase response in delirium. J Psychosom Res 2007;62:521-5.

85 van Munster BC, Korevaar JC, Zwinderman AH, et al. Time-course of cytokines during delirium in elderly patients with hip fractures. J Am Geriatr SoC 2008;56:1704-9.

86 Adamis D, Treloar A, Martin FC, et al. APOE and cytokines as biological markers for recovery of prevalent delirium in elderly medical inpatients. Int J Geriatr Psychiatry 2007:22:688-94

87 Clough Z, Jeyapaul P, Zotova E, et al. Proinflammatory Cytokines and the Clinical Features of Dementia With Lewy Bodies. Alzheimer Dis Assoc Disord, 2013. Jun 20. [Epub ahead of print] www.ncbi.nlm.nih.gov/pubmed/23792923

88 Holmes C, Cunningham C, Zotova E, et al. Proinflammatory cytokines, sickness behavior, and Alzheimer disease. Neurology 2011;77:212-18. 
89 Rognve A, Auning E, Fladby T, et al. The pre-dementia stage of dementia with Lewy bodies. Alzheimers Demen 2011;7:S749-50.

90 Gaig C, Valldeoriola F, Gelpi E, et al. Rapidly progressive diffuse Lewy body disease. Mov Disord 2011:26:1316-23.

91 Jicha GA, Schmitt FA, Abner E, et al. Prodromal clinical manifestations of neuropathologically confirmed Lewy body disease. Neurobiol Aging 2010;31:1805-13.
92 Lundstrom $\mathrm{M}$, Edlund $\mathrm{A}$, Bucht $\mathrm{G}$, et al. Dementia after delirium in patients with femoral neck fractures. J Am Geriatr Soc 2003;51:1002-6.

93 Kat MG, Vreeswijk R, de Jonghe JFM, et al. Long-term cognitive outcome of delirium in elderly hip surgery patients. A prospective matched controlled study over two and a half years. Dement Geriatr Cogn Disord 2008;26:1-8.

94 Fong TG, Jones RN, Shi P, et al. Delirium accelerates cognitive decline in Alzheimer disease. Neurology 2009;72:1570-5. 